\title{
Impact of Self Stigma on Quality of Life of People with Mental IIIness at Dilla University Referral Hospital, South Ethiopia
}

\author{
Alem Eskeziya Ayenalem ${ }^{1, *}$, Tenaw Yimer Tiruye ${ }^{2}$, Muhammed Seid Muhammed $^{3}$ \\ ${ }^{1}$ Department of Psychiatry, College of Health Science and School of Medicine, Dilla University, Dilla, Ethiopia \\ ${ }^{2}$ Public health Department, College of Health Science, Debre Markos University, Debre Markos, Ethiopia \\ ${ }^{3}$ Department of Psychiatry, College of Medicine and Health Science, Wollo University, Dessie, Ethiopia
}

Email address:

alemayenalem123@gmail.com (A. E. Ayenalem), tenyim09@gmail.com (T. Y. Tiruye), muhamedseid@yahoo.com (M. S. Muhammed) ${ }^{*}$ Corresponding author

\section{To cite this article:}

Alem Eskeziya Ayenalem, Tenaw Yimer Tiruye, Muhammed Seid Muhammed. Impact of Self Stigma on Quality of Life of People with Mental Illness at Dilla University Referral Hospital, South Ethiopia. American Journal of Health Research. Vol. 5, No. 5, 2017, pp. 125-130. doi: 10.11648/j.ajhr.20170505.12

Received: July 25, 2017; Accepted: August 7, 2017; Published: August 25, 2017

\begin{abstract}
The stigma associated with mental illness can potentially be a reason for lower quality of life among persons with mental illness. Since self stigma can also exist without actual stigma from the public, more hidden and inside, it seems to be the worst form of stigma against people with mental illness and can directly affect the overall well being of people with mental illness. Moreover, it is also a great barrier for social interaction. However, no study is available on impact of self stigma on quality of life of people with mental illness in South Ethiopia generally and particularly in Dilla town. The purpose of this study was to assess the impact of self stigma on quality of life of people with mental illness at Dilla University Referral Hospital, south Ethiopia. The study was conducted in the psychiatry outpatient department of Dilla University referral hospital. An institutional based cross-sectional study was conducted on 317 people with mental illness that attended psychiatric clinic for mental health service from June $1^{\text {st }}$ to $30^{\text {th }}, 2016$. Data were collected using a pre tested interviewer administered structured questionnaires. Self-stigma was measured using Internalized Stigma of Mental Illness (ISMI) Scale which was validated and used in many other similar settings. Quality of life was measured using quality of life assessment tool Short Form, Version-2 (SF-36). In this study, the prevalence of self stigma among people with mental illness was $32.1 \%$. Regarding Quality of life (QoL) of people with mental illness, 54.6\% were experiencing lower quality of life. As self stigma increases by one unit in studied participants, quality of life decreases by $4.1 \%$ (OR=0.041; $95 \%$ C.I: $-0.065,-0.012)$. In other words, self stigma and QOL were inversely correlated( $\mathrm{r}=-0.076)$ which indicates that stigma worsen $\mathrm{QoL}$ of people with mental illness. The result of this study was revealed that, apparently high prevalence of self-stigma among persons with mental illness and lower quality of life. To improve QoL of people with mental illness, tackling stigma need to be addressed by health professionals, community, researchers, policy makers and stakeholders.
\end{abstract}

Keywords: Impact, Self Stigma, Quality of Life, Mental Illness

\section{Introduction}

Worldwide around 450 million people are suffering from mental disorders and it is associated with high disease burden, disability and premature death [1-2].

People with mental illness (PWMI) have double problem facing them associated with their illness. These include stigma. Stigma is mark (labeling) to one based on his/her psychiatric illness or taking psychotropic medication [3]. Stigma results from a process by which certain individuals (groups) unjustifiably are rendered shameful, excluded \& discriminated [4]. The prevalence of self stigma among psychiatric clients is high and it affects lives of people with mental illness in multidimensional ways [2, 4 \& 5].

In the studies done in 3 selected psychiatric hospitals in 
Iran, Switzerland, European commission and Athens University Greece showed that self stigma is high (40\%) and due to this high magnitude of stigma, PWMI were excluded from work opportunities, employers were unwilling to hire PWMI. Reduced personal \& family income due to low wage as they hired by discount due to their status of illness was a burden. Stigma \& discrimination worsen mentally illness and leads to social exclusion, inability to participate in important life activities and poor tendency to seek treatment which last hampers one major dimension of QoL [6-10]. The possible factors were socio demography \& diagnosis, education, lack of support and religion as major influencing factor of self stigma. The studies reported that due to self stigma held by PWMI, there is poor treatment behavior, high prevalence of disability and economic burden which leads to social exclusion, inability to participate in important life activities and poor tendency to seek treatment that impede treatment and non adherence which last hampers one major dimension of QoL [8, $9 \& 11]$. A study in the capital city of Ethiopia on outpatients with schizophrenia and in Jimma University Specialized Hospital (JUSH) Psychiatry clinic using the same scale also reported high prevalence of internalized stigma [12-13]. In the above study, patients who were living in rural areas were more likely to exhibit higher internalized stigma than urban residents and those with high income. From all identified stigmatized beliefs, self stigma is more deleterious [12].

Self Stigma is an exacerbating factor for mental illness and it affects lives of PWMI including poor help seeking behavior, social exclusion, being unemployed, economical \& social ruin and premature death [3, 14]. The possible factors for high prevalence of stigma are ignorance, fear of injury, derived by social conformity, internalization, cultural facts about causation of mental illness, actual (perceived) absence of treatment and preventive modalities and lack of information system to educate professional and general population [4]. In addition to high self stigma people with mental illness has also poor quality of life. A study done on schizophrenic patient in Egyptian psychiatry and addiction hospital showed that patients have poor quality of life before nursing intervention [15]. Although some effort tried to reduce stigma against PWMI, stigmatization \& discrimination toward PWMI, it is still persistent.

In Ethiopia, there is widespread mental illness and associated deep-rooted stigma towards PWMI [1, 14] but few studies have done on level of self stigma of PWMI and its impact on lives in SNNPR, Ethiopia generally and in Dilla specifically where the magnitude of mental illness and substance use are highly prevalent. In addition to this, the level and correlates of self stigma among psychiatric patients in south Ethiopia particularly in Dilla University Referral Hospital (DURH) Psychiatry clinic clients has not been investigated.

\section{Methods}

The study was conducted from June - October 2016 at
Dilla University Referral Hospital (DURH). An institution based cross-sectional study was carried out on 317 mentally ill patients by using consecutive sampling techniques who were visiting DURH psychiatry clinic. The sample size was determined using single population proportion formula by using $25.12 \%$ level of self stigma [16]. Respondents were screened using the Clinical Global Impression (CGI) scale to assess their eligibility to participate in the interview for the study [17]. The scale assesses the degree of the severity of the patients' mental illness, response from mental illness and therapeutic efficacy and side effects of drugs. New patients were screened only for the severity of their illness. Patients who were severely psychotic, catatonic, incoherent and too disorganized to participate in the interviews of the study were excluded. Therefore, patients involved in the study were only those who were above 18 years old and rated with at least a less severe state of mental illness, on remission and good efficacy index by the psychiatry nurses.

Data was collected using standard questionnaire for assessing self stigma that adapted from the reviewed literatures. Data was collected by trained psychiatric nurses through interviewer-administered structured questionnaires and a patient chart review to identify other medical information. The data collection was supervised by specialist mental health workers. Data collectors and the supervisors were trained on the contents and procedures of the data collection for two consecutive days.

The Internalized Stigma of Mental Illness (ISMI) Scale [18] was used to measure self stigma. The scale has been used in several studies [19-20]. The ISMI scale have a total of 29 items on a 4-point Likert scale (1=strongly agree to $4=$ strongly disagree) measure containing five subscales. The overall score was obtained by summing the scores of the five subscales. Higher score showed higher self-stigma. A study in Iran showed that the ISMI subscales had reliability values (Cronbach's alpha) of (alienation $=0.84$, stereotype endorsement $=0.71$, discrimination experience $=0.87$, social withdrawal $=0.85$ and stigma resistance $=0.63$ ). In the current study, the following reliability values (Cronbach's alpha) were found: alienation $=0.84$, stereotype endorsement $=0.73$, discrimination experience $=0.79$, social withdrawal $=0.77$, stigma resistance $=0.65$, over all self stigmas $=0.89$. For QOL assessment the Medical Outcomes Study Short Form 36, Version 2 (SF-36) was used [18, 20]. The SF-36 has been utilized in monitoring health out- comes in patients with a variety of illnesses including mental illness as well as a wide range of diseases and chronic conditions. It is scale of 36 items with eight domains and in each domain certain types of questions from the 36s are included [19-20]. Cultural validity and reliability of items on the tool was done in Ethiopia on eight sub domains. The domains are: Physical Functioning (SF), Role Impairment due to Physical Problem (RIPP), Role Impairment due to Emotional Problems (RIEP), Bodily Pain (BP), Vitality (V), Societal Functioning (SF), Mental Health (MH) and General Health Perception (GHP). In this survey, item internal consistency was high (Cranach's alpha $>0.7$ ) and item discriminate variability was low for all 
items except for vitality which varied between 0.28 and 0.61 [21].

To increase the quality of data, the questionnaire was translated to Amharic \& Gediofa and then back to English by using different translators to keep the consistency of the questionnaire and necessary correction was made. The collected data were double entered using EPI data software version 3.1. Then it was exported to SPSS version 20.0 for data processing and analysis. The following variables were measured as follows during analysis: (a) High self stigmaimplies Psychiatric patients who have scored above or equal of 2.5 in four likert scale questions of ISMI. (b) Lowself stigma- implies Psychiatric patients who have scored below 2.5 in four likert scale questions of ISMI. (c) Past many admission=three and more times hospitalized in psychiatric clinic. (d) Co-morbidities = physical illness that occur simultaneously with mental illness. Descriptive tests like proportions mean and standard deviations and analytic tests like bivariate and multivariate logistic regression analysis were computed. Odds ratio along with the 95\% CI was estimated to ascertain the association between covariates and self stigma. Covariates that have $P$ value of $<0.25$ at the bivariate analysis were included in the multivariate logistic regression to control all possible confounding factors. For all statistical tests, $P$ value $\leq 0.05$ was used as a cut-off point for statistical significance.

Officially written approval letter was obtained from Research and Dissemination Office of Dillaand DURH Psychiatry clinic. Written informed consent was also obtained from each study participant.

\section{Results}

\subsection{Socio-demographic Characteristics of Respondents}

Of the total 317 respondents 119 (56.5\%) were urban residents with a mean age of $33.60 \pm 9.59$ years. The majority, $220(69.4 \%), 141(44.6 \%)$ and $145(45.7 \%)$ of the study participants were males, Gedio by ethnicity and protestant by religion respectively. Concerning marital status, more than halve $(51.3 \%)$ of the studied participants were married followed by single $(47.6 \%)$. The data also revealed that, 116 $(36.3 \%)$ of the participants were completed elementary school. Regarding to occupational status, 121 (38.1\%) of the studied participants were farmers (Table 1).

Table 1. Socio-demographic characteristics of PWMI at DURH, South Ethiopia, $2016(n=317)$.

\begin{tabular}{ll}
\hline Variable & Frequency $(\%)$ \\
\hline Sex & $220(69.4)$ \\
Fale & $97(39.7)$ \\
Age(in years) & \\
$18-24$ & $67(21)$ \\
$25-34$ & $110(34.8)$ \\
$35-49$ & $140(44.3)$ \\
Ethnicity & \\
Gedio & $141(44.6 \%)$ \\
\hline
\end{tabular}

\begin{tabular}{ll}
\hline Variable & Frequency (\%) \\
\hline Sex & $76(24 \%)$ \\
Sidamo & $47(14.8 \%)$ \\
Guragie & $36(11.8 \%)$ \\
Oromo & \\
Peligion & $145(45.7)$ \\
Orotestant & $86(27.1)$ \\
Marital status & \\
Single & $132(41.6)$ \\
Married & $151(47.5)$ \\
Others & $34(10.9)$ \\
Educational status & \\
Illiterate & $75(23.6)$ \\
Elementary & $116(36.3)$ \\
High school & $44(14.0)$ \\
College and above & $82(25.9)$ \\
Occupation & \\
Unemployed & $58(18.4)$ \\
Student & $27(8.4)$ \\
Farmer & $121(38.1)$ \\
Governmental worker & $111(35.1)$ \\
\hline
\end{tabular}

Concerning family support of study participants, more than three-quarters $257(81.1 \%)$ received support from the family but the rest did not get any support. With regard to social support, the majority of the studied participants $162(51.1 \%)$ did not have any social support (Table 2).

Table 2. Supporting system for study participants at DURH, South Ethiopia, $2016(n=317)$.

\begin{tabular}{|ll}
\hline Variable & Frequency (\%) \\
\hline Living with & $68(21.4)$ \\
\hline Alone & $221(69.6)$ \\
With family & $23(7.2)$ \\
With relatives & $5(1.8)$ \\
With friend & \\
Support from family & $257(81.1)$ \\
Yes & $60(18.9)$ \\
No & $104(32.8)$ \\
Type of support from family & $81(25.4)$ \\
Moral support & $61(19.4)$ \\
Physical support & $71(22.4)$ \\
Financial support & \\
Food support & $155(48.9)$ \\
Support from societal area & $162(51.1)$ \\
Yes & \\
No & $64(20.8)$ \\
Societal group who gave support & $42(13.3)$ \\
From friends & $15(4.6)$ \\
From relatives & $23(7.4)$ \\
From religion & $9(2.8)$ \\
From government & \\
From neighbours & \\
\hline
\end{tabular}

\subsection{Diagnosis}

The majority of the patients were diagnosed with psychosis $165(52.05 \%)$ followed by mood disorders 136(43.02\%) (table 3). 
Table 3. Diagnosis of study participants at DURH, South Ethiopia, 2016 $(n=317)$.

\begin{tabular}{ll}
\hline Variable & Frequency (\%) \\
\hline Psychosis & $165(52.5)$ \\
Mood disorder & $136(43.02)$ \\
Anxiety disorder & $10(3)$ \\
Others & $6(1.95)$ \\
\hline
\end{tabular}

Beside their psychiatric diagnosis, 36 (11.40\%) had comorbidities and 205(64.6\%) reported some kind of side effects of their medication. Of those who had drug side effects, $88(27.9 \%)$ were reported weight gains. The mean time since the onset of the patients' mental illness was 4.37 $(\mathrm{SD}=2.83)$ years while the mean time since the start of medical follow up was $3.43(2.26 \%$ ) years (table 4$)$.

Table 4. Medication related characteristics of study participants at DURH, South Ethiopia, $2016(n=317)$.

\begin{tabular}{ll}
\hline Variable & FREQUENCY (\%) \\
\hline Duration of treatment & \\
\hline$\leq 5$ years & $153(48.3)$ \\
$6-10$ years & $110(34.7)$ \\
$>10$ years & $54(17.6)$ \\
experience any side effect associated with medication(antipsychotics, \\
antidepressants and benzodiazepines) & \\
Yes & $205(64.6)$ \\
No & $112(35.4)$ \\
Types of side effect & \\
\hline
\end{tabular}

\begin{tabular}{ll}
\hline Variable & FREQUENCY (\%) \\
\hline Duration of treatment & \\
\hline Weight gain & $88(27.9)$ \\
Tremor & $43(13.7)$ \\
Sexual problem & $59(18.5)$ \\
Sedation & $6(1.8)$ \\
Gait abnormality & $4(1.2)$ \\
Others & $3(0.9)$ \\
\hline
\end{tabular}

\subsection{Magnitude of Self Stigma and Level of Quality of Life}

From the study participants, $32.1 \%$ had high level of selfstigma based on total stigma score. The mean score for the overall self stigma was 2.26 with standard deviation of 0.39 .

With regard to level of quality of life, more than halve (53.1\%) of the study participants had low quality of life in area of physical domain and $46.8 \%$ had role impairment of physical problem while $45.6 \%$ and $36.7 \%$ of the studied participants experienced low quality of life due to role impairment of emotional problem and pain experience, respectively. In addition, $55.8 \%, 61.7 \%$ and $50.7 \%$ of the studied participants were also exhibiting low quality of life in social aspect, in respect to energy, and social life respectively. Greater than half (51.6\%) of the studied participants had low quality of life related to general health (Figure 1). Over all, more than half of the studied participants $(54.6 \%)$, had lower than the mean QoL score $(\leq 65.5)$.

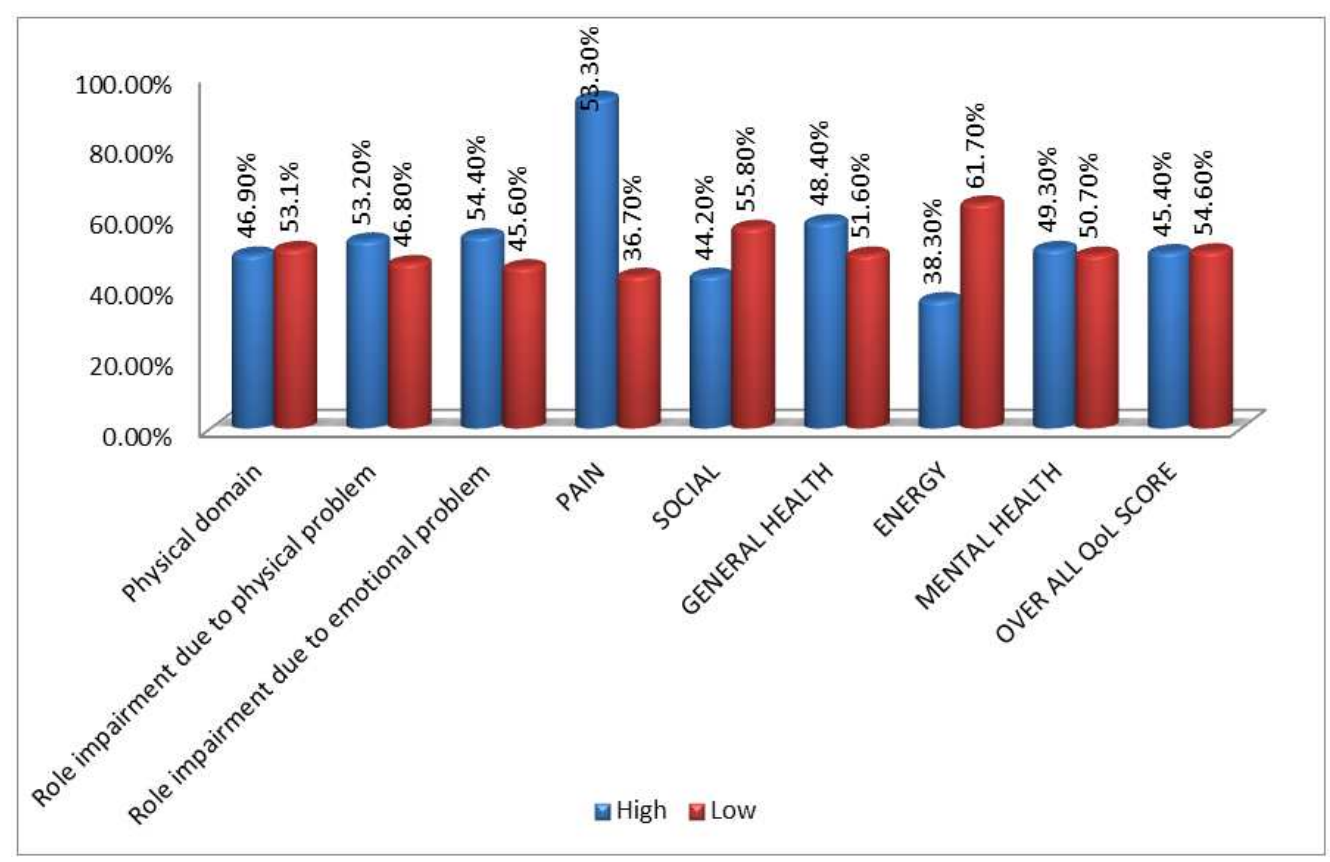

Figure 1. Quality of life of the study participants at DURH, South Ethiopia, 2016.

\subsection{Relationship Between Self Stigma and Quality of Life}

As stigma increases by one unit in studied participants of PWMI, quality of life decreases significantly by $4.1 \%(\beta 1=-$ 0.041, 95\% C.I:-0.065,-0.012). In this study self-stigma and Quality of life were inversely correlated $(\mathrm{r}=-0.076)$ which indicates stigma worsen QoL of people with mental illness (Table 5).
Table 5. Correlation between QoL and self stigma of studied participants at DURH, South Ethiopia, $2016(n=317)$.

\begin{tabular}{lllll}
\hline \multirow{2}{*}{ StudyVariables } & QoL & \multicolumn{3}{l}{ Stigma } \\
\cline { 2 - 5 } & R & P & R & P \\
\hline Sol & - & - & -0.041 & 0.707 \\
Stigma & -0.041 & 0.707 & - & - \\
\hline
\end{tabular}




\section{Discussion}

Regarding the overall quality of life, a high percentage of the studied participants demonstrated low level of quality of life. This is in line with study done in turkey in 2010 [7] with the overall mean QoL score of patients was 61.5 (range 24.6 - 89.6, SD 17.4) suggesting that patients' QoL was impaired. This result is also supported by a study done in Ethiopia which showed that epileptic patient's co morbid with anxiety and depression had poor quality of life [22]. The finding is also revealed that there was high prevalence of self-stigma which was $32.1 \%$. This result is consistent with the findings of the study on self-stigma among people with mental illness $\mathrm{s}$ in southern west Ethiopia in 2013 [16]. This finding is also supported by study done in Europe which indicated that there is rampant self-stigmatization and discrimination in different areas of life activities due to one or more severe mental illness in 2002 [9]. But the finding of this study is incongruent with findings of previous studies done in Iran, Nigeria and India [14, 23 \& 24]. This inconsistent finding may be happened due to different in severity of mental illness since the current study have been done on all mental illness which included less severe form of mental illness while the formers have been done on severe form of mental illness which stigma is considered higher than that of less severe form of mental illness. Considering relationship between stigma and quality of life of PWMI, it was explained that self stigma eroded quality of life of PWMI, As stigma increases by one unit, quality of life decreases by $4.1 \%$ (Std. $\beta=-0.041$, 95\% C.I: $-0.065,0.012$ ). In this study self-stigma and QoL were inversely correlated $(\mathrm{r}=-0.076)$ which indicates stigma worsen QoL of people with mental illness.

The finding of this study did support that self stigma has significant negative impact on quality of life of people with mental illness which was congruent with findings of study done in Cairo in 2012 [25], that reported stigma is negatively associated with quality of life. This finding is also in line with some other studies [26-27]. Lastly the finding of this study indicates high prevalence of self stigma among people with mental illness which is incongruent with findings of plenty of studies. This contradict may be due to there is better stigma reduction strategies and better awareness about mental illness there than in the setting of this study.

\section{Conclusion and Recommendation}

The result of this study revealed that relatively high prevalence of self-stigma among people with mental illness. Self stigma was negatively associated with quality of life. So, great efforts have to be invested on stigma reduction strategy among people with mental illness at both clinical and community settings which could empower people with mental illness towards stigma so as to improve the life of people with mental illness. Psychiatric institutions in collaboration with Ministry of health should do more roles not only in medical management of patients but also in promotion of their social life. Further study on self esteem as predictors of self stigma and quality of will be recommended.

\section{List of Acronyms/Abbreviations}

\author{
BP: Bodily Pain \\ CGI: Clinical Global Impression \\ DALY: Disability Adjusted Life Years \\ DURH; Dilla University Referral Hospital \\ GHP: General Health Perception \\ ISMH: Internalized Stigma of Mental Illness \\ JUSH: Jimma University Specialized Hospital \\ MH: Mental Health \\ PF: Physical Functioning \\ PWMI: People with Mental Illness \\ QoL: Quality of Life \\ RIEP: Role Impairment due to Emotional Problem \\ RIPP: Role Impairment due to Physical Problem \\ SF: Societal Functioning \\ SF-36: Short Form tool for measuring of Quality of life \\ SNNPR: Southern Nations, Nationalities and People's \\ Region \\ V: Vitality \\ WHO: World Health Organization \\ WHOQOL: World Health Organization Quality Of Life
}

\section{Acknowledgements}

The authors would like to thank Dilla University, Data collectors for their responsible data collection and individuals who were helping the authors in manuscript preparation.

\section{References}

[1] Woldegiworgise T, Simme K: National mental health strategy, Ethiopia: Federal ministry of Health; 2012/13.

[2] World Health Organization (WHO): Depression as a global crisis: World mental health day. 2012.

[3] Daneil E, Marilyn F, Efra G: Stigma \& Help seeking for mental health among college students: Medical care research \& review. 2009.

[4] World Health Organization (WHO): Reducing stigma \& discrimination against older people with mental disorders. Geneva, Switzerland: World Health Organization Press. 2002.

[5] World Health Organization (WHO): Investing in mental health. Geneva, Switzerland: World Health Organization Press. 2003.

[6] Patrick JM, Marcelino L, Nicolas R: Constructs \& concepts comprising the stigma of mental illness. Psychology, Society \& Education 2012, 4(2): 183-194.

[7] Pitkanen A, Anneli: Improving quality of life of patients with schizophrenia. In acute psychiatric wards. 010.

[8] World Health Organization (WHO): Mental health and stigma, a call for action by world health ministries. Geneva, Switzerland: World Health Organization Press. 2001. 
[9] Mc Daid, David: Countering the stigmatization \& discrimination of people with mental health problems in Europe: European commission. 2008.

[10] Helia G, Marzih N, Lars J: Internalized stigma of mental illness. Stigma research \&action 2011, 1(1): 11-17.

[11] Aphroditiz S, Michael M: Stigma related to help seeking from mental health professionals. Health Science Journal 2010, 4(2): 77-83.

[12] Girma E, Tesfaye M, Froseschl G, Norbert Moller-Lekimku AM, Muller N, Dehning S: Publicstigma against people with mental illness in Gilgel Gibe field research center (GGFRC)in south west Ethiopia. PLOS One 2013, 8(12): 1-9.

[13] Assefa D, Shibre T, Asher L, Fekadu A: Internalized stigma among patients with schizophrenia in Ethiopia: a crosssectional facility-based study. BMC Psychiatry 2012, 12: 239.

[14] Faith BD, Jawel S, Andrea E: Experience of stigma among out patients with schizophrenia. Schizophrenia bulletin 2002, 28(1): 143-155.

[15] Sabah M E, Faten H A: Effectiveness of Psychiatric Nursing Intervention on Adherence to Medications and Quality of Life of Schizophrenic Patients. American Journal of Nursing Science 2016, 5(6): 232-239.

[16] Girma E, Tesfaye M, Guenter F, Sandra D, et al: Facility based cross-sectional study of self stigma among people with mental illness: towards patient empowerment approach. International Journal of Mental Health Systems 2013, 7: 21.

[17] Joan B, Steven DT: The Clinical Global Impressions Scale: Applying a Research Tool in Clinical Practice. Psychiatry 2007, 4(7): 28-37.

[18] Dan S, Mihae M: Gender disparities in mental health. Journal of affective disorder 2005, 86(2): 205-213.
[19] Mittal D, Sullivan G, Chekuri L, Allee E, Corrigan PW: Empirical studies of self-stigma reductionstrategies: A critical review of the Literature. Psychiatrserv 2012, 63(10): 974-981.

[20] Kebede D, Alem A, Negash A, Shibrie T, etal: Health related quality of life (SF-36) survey in Butajira, rural Ethiopia: Normative data and evaluation of reliability and validity. Ethiopian medical journal 2004, 42(4): 289-297.

[21] Aloba O, FatoyeO, Mapayi B, Akinsulore S: A review of Quality of Life studies in Nigerian patients with psychiatric disorders. Afr J Psychiatry 2013, 16(5): 333-337.

[22] Minale TT, Niguse YM, Teketel TW, Andargie AA, Tilahun BM, Molla AY: Assessment of quality of life and associated factors among people with epilepsy attending at Amanuel Mental Specialized Hospital, Addis Ababa, Ethiopia. Science Journal of Public Health 2014, 2(5): 378-383.

[23] Ehab SR, Wessam AE: Relation between insight \& quality of life in patients with schizophrenia. Current psychiatry 2010, 17(3): 43-48.

[24] West ML, Yanos PT, Smith SM, Reo D, Lysaker PH: Prevalence of Internalized Stigma among Persons with Severe Mental Illness. Stigma Res action 2011, 1(1): 3-10.

[25] Omnia MA, Enayat AEW, Zeinab AEH, et al: The Effect of Stigma on Quality of Life among People with Mental Illnesses. 2015.

[26] Amin M, AbdelAziz HE, Lawindy M: Schizophrenic Patients Families Psycho-Education: Outcomes on Patient Quality Of Life and Disease Relapse Rate. Egyptian Journal of Psychiatry 2004, 1(23): 59-74.

[27] James L: Self stigma and quality of life among people with mental illness who receive compulsory treatment. Journal of community psychology 2012, 40 (6): 699-714. 